PROCEEDINGS OF THE

AMERICAN MATHEMATICAL SOCIETY

Volume 135, Number 8, August 2007, Pages 2567-2571

S 0002-9939(07)08781-3

Article electronically published on March 22, 2007

\title{
MATRIX COEFFICIENTS AND COADJOINT ORBITS OF COMPACT LIE GROUPS
}

\author{
A. H. DOOLEY AND R. W. RAFFOUL
}

(Communicated by Jane M. Hawkins)

\begin{abstract}
Let $G$ be a compact Lie group. We use Weyl functional calculus (Anderson, 1969) and symplectic convexity theorems to determine the support and singular support of the operator-valued Fourier transform of the product of the $j$-function and the pull-back of an arbitrary unitary irreducible representation of $G$ to the Lie algebra, strengthening and generalizing the results of Cazzaniga, 1992. We obtain as a consequence a new demonstration of the Kirillov correspondence for compact Lie groups.
\end{abstract}

\section{INTRODUCTION}

Let $G$ be a tame unimodular Lie group, $\mathfrak{g}$ its Lie algebra and $\mathfrak{g}^{*}$ the vector space dual of $\mathfrak{g}$. The group $G$ operates on $\mathfrak{g}^{*}$ by the coadjoint action yielding, according to the Kirillov-Kostant philosophy, a consequent parametrization of the unitary irreducible representations of $G$ by orbits satisfying a certain integrality condition, equivalent to the following character formula: let $\pi$ be a finite-dimensional unitary irreducible representation of $G$ related to the coadjoint orbit $\mathcal{O}$ - for almost all $\pi$ in the reduced dual of $G$, as an equality of distributions,

$$
j(X) \operatorname{Tr} \pi(\exp (X))=\int_{\mathcal{O}} e^{i \eta(X)} d \mu_{\mathcal{O}}(\eta)
$$

for all $X \in \mathfrak{g}$ in a sufficiently small neighbourhood of 0 , where $\mu_{\mathcal{O}}$ is a Liouville measure on $\mathcal{O}$ and the $G$-invariant function $j$ is the analytic square root of the jacobian of the exponential map, $j(0)=1$.

When $G$ is compact, (1.1) follows from the Weyl character formula and a wellknown result [7, Theorem 2] of Harish-Chandra (see [15] or [12 for an exposition). It holds as an identity over all of $\mathfrak{g}$, and a unitary irreducible representation of highest weight $\lambda$ corresponds to the orbit though $\lambda+\delta$, where $\delta$ is half the sum of the positive roots.

This was originally proved by Kirillov in [10, where the validity of formula (1.1) for nilpotent groups was also demonstrated and its universality conjectured. It has since been verified for many other classes of Lie groups, notable contributions being [16] and [9].

Received by the editors April 18, 2006

2000 Mathematics Subject Classification. Primary 43A77, 22E99; Secondary 47Nxx.

Key words and phrases. Coadjoint orbits, Lie groups, matrix coefficients, moment map, Weyl functional calculus.

The authors gratefully acknowledge the support of the Australian Research Council.

(C)2007 American Mathematical Society 
It is remarkable in particular that the support of the Fourier tranform of the product $j(X) \operatorname{Tr} \pi(\exp (X))$ is a single integral coadjoint orbit. When $G=S U(2)$, on extending the Kirillov formula to the matrix coefficients, Cazzaniga [4 makes the interesting observation that the support and singular support of the Fourier transform of an arbitrary trigonometric polynomial multiplied by $j$ also have descriptions in terms of the coadjoint orbit picture. We extend his results to the case of an arbitrary semisimple compact Lie group.

Fixing a Cartan subalgebra $\mathfrak{t} \subseteq \mathfrak{g}$, let $\mathcal{O}_{\lambda}$ be the coadjoint orbit through $\lambda \in \mathfrak{t}^{*}$ and let $\mu_{\lambda}$ be the Liouville measure on $\mathcal{O}_{\lambda}$; choose an ordered basis $x=\left(x_{1}, \ldots, x_{n}\right)$ of $\mathfrak{g}$ which is orthonormal with respect to the Killing form and let $W$ be the Weyl group. We have the following

Theorem 1.1. Let $G$ be a compact, semisimple, connected Lie group and let $\pi$ be a unitary irreducible representation of $G$ with highest weight $\lambda$. The operatorvalued Fourier transform of $j(X) \pi(\exp (X))(X \in \mathfrak{g})$ has support contained in the convex hull of $\mathcal{O}_{\lambda+\delta}$ and singular support equal to $\bigcup_{w \in W} \mathcal{O}_{\lambda+w \cdot \delta}$; furthermore, its entries are, in the sense of distributions, polynomial in the differential operators

$\frac{\partial}{\partial x_{1}}, \ldots, \frac{\partial}{\partial x_{n}}, x \cdot \frac{\partial}{\partial x}$ applied to the measure $\mu_{\delta} * I_{\lambda}$, where $I_{\lambda}$ is the canonical measure on the moment set of $\pi$.

Here the "moment set" of a Lie group representation is the image of the symplectic geometry moment map of the Hamiltonian action of $G$ ([18, Proposition 1.1]) on the projectivisation of its representation space, which is afforded a symplectic structure [3, p. 345].

We remark that in [5] the first author and N.J. Wildberger produce character formulae generalizing (1.1) by wrapping central distributions supported on $\bigcup_{w \in W} \mathcal{O}_{\lambda+w \cdot \delta}$.

It follows from the arguments of [2, Lemme 15] (and in more generality in [13, Proposition VIII.1.30(i)]) that the set of extremal points of the convex hull of $\mathcal{O}_{\lambda+\delta}$ is the orbit $\mathcal{O}_{\lambda+\delta}$. In view of this, the second statement in the above theorem can be regarded as a new demonstration of the Kirillov correspondence for compact Lie groups.

By means of a new estimate for the singular support of the $j$-modified Fourier transform of the pull-back character, our results also yield a short route to the Kirillov formula (1.1) from the work of Harish-Chandra, independent of the Weyl character formula. Details will appear in the second author's Ph.D. thesis.

The authors would like to thank B. Jefferies and N.J. Wildberger for their invaluable advice.

\section{Proof of the THEOREM}

With $x=\left(x_{1}, \ldots, x_{n}\right)$ a basis of the Lie algebra $\mathfrak{g}$ as above, write $X=\xi \cdot x$ $\left(\xi \in \mathbb{R}^{n}\right)$ for arbitrary $X \in \mathfrak{g}$ and note that

$$
\pi(\exp (X))=e^{\xi \cdot d \pi(x)}
$$

where $d \pi(x):=\left(d \pi\left(x_{j}\right)\right)_{j}$.

Since the $n$-tuple $d \pi(x)$ consists entirely of skew-hermitian matrices, the Fourier transform $\left(e^{\xi \cdot d \pi(x)}\right)^{\wedge}$ is given by Nelson's formula [14] which in the notation of [8] 
is

$$
\begin{aligned}
&\left(e^{\xi \cdot d \pi(x)}\right)^{\wedge}= \sum_{k=0}^{d_{\lambda}} \sum_{j=0}^{d_{\lambda}-k-1} \sum_{m=0}^{j}(-1)^{k} A_{j, m}^{(n)}\left(d \pi(x) \cdot \frac{\partial}{\partial x}\right)^{k} \\
& \cdot \phi_{n-j-k-1}\left(d \pi(x) \cdot \frac{\partial}{\partial x}\right)\left(\frac{\partial}{\partial x} \cdot x\right)^{m} \mu_{d \pi(x)}
\end{aligned}
$$

where

$$
A_{j, m}^{(n)}:=(-1)^{m}\left(\begin{array}{c}
j \\
m
\end{array}\right) \frac{i^{-n+j+1}}{(n-1-j+m) !},
$$

$d_{\lambda}:=\operatorname{deg} \pi, \phi_{j}$ is the sum of the principal minors of order $j$ of the matrix $d \pi(x)$. $\frac{\partial}{\partial x}:=\sum_{j} d \pi\left(x_{j}\right) \frac{\partial}{\partial x_{j}}$ and $\mu_{d \pi(x)}:=\nu \circ \Psi_{\pi}^{-1}, \nu$ being a unitarily invariant probability measure on $\Omega:=\left\{z \in \mathbb{C}^{n}:|z|=1\right\}$ and

$$
\Psi_{\pi}(u):=\frac{1}{i}\left(\left\langle d \pi\left(x_{1}\right) u, u\right\rangle, \ldots,\left\langle d \pi\left(x_{n}\right) u, u\right\rangle\right)
$$

for $u \in \Omega$, where $\langle\cdot, \cdot\rangle$ is the usual inner product on $\mathbb{C}^{n}$, is the moment map of $\pi$ [18.

By commutation relations, $\frac{\partial}{\partial x} \cdot x=x \cdot \frac{\partial}{\partial x}+n I$, where $I$ is the identity operator, and since the Fourier transform of the $j$-function is $\mu_{\delta}$ (from (1.1) with $\lambda=0$ ) the second statement follows.

From the results [2] and [18, when the set $\{\lambda-w \cdot \lambda: w \in W\}$ contains no roots, the moment set $I_{\lambda}$ is the convex hull of $\mathcal{O}_{\lambda}$, and furthermore the projection of $I_{\lambda}$ onto $t^{*}$ is the convex hull of the Weyl orbit of $\lambda$. Similarly, by Kostant's convexity theorem [11, the projection of $\mathcal{O}_{\delta}$ onto $\mathfrak{t}^{*}$ is the convex hull of the Weyl orbit of $\delta$. Hence,

$$
\operatorname{supp} \mu_{\delta} * I_{\lambda} \subseteq \operatorname{conv} \mathcal{O}_{\lambda+\delta}
$$

and

$$
\operatorname{singsupp} \mu_{\delta} * I_{\lambda}=\bigcup_{w \in W} \mathcal{O}_{\lambda+w \cdot \delta} .
$$

On the other hand, when the set $\{\lambda-w \cdot \lambda: w \in W\}$ contains a root, or equivalently, a simple root, $I_{\lambda}$ is properly contained in the convex hull of $\mathcal{O}_{\lambda}$, but the extremal set of $I_{\lambda}$ includes $W \cdot \lambda$, and the line joining $w^{\prime} \cdot \lambda$ and $w^{\prime \prime} \cdot \lambda$ $\left(w^{\prime}, w^{\prime \prime} \in W\right)$ is contained in $I_{\lambda}$ whenever the difference $w^{\prime} \cdot \lambda-w^{\prime \prime} \cdot \lambda$ is not a root; in fact the whole convex hull of the set of weights for which the pairwise difference is not a root is contained in $I_{\lambda}$ (see [17, Lemma 7.1]). Hence the theorem follows as before, making use of the fact that the intersection of the image of the moment map with the positive Weyl chamber is a convex polytope [6].

\section{The example of $S U(2)$}

We identify the Lie algebra $\mathfrak{s u}(2)$ with $\mathfrak{s u}(2)^{*}$ by the trace form $X \longleftrightarrow-\frac{1}{2} \operatorname{Tr}(X \cdot)$. Noting that $-\frac{1}{2} \operatorname{Tr}\left(X^{2}\right)=\operatorname{det} X$, we have by invariance that the coadjoint orbits of $S U(2)$ are spheres. We write $B_{n}$ for the the solid ball of radius $n$ in $\mathbb{R}^{3} \approx \mathfrak{s u}(2)$.

The irreducible representations of $S U(2)$ are parameterized by the set $\mathbb{N}$. The moment set $I_{n}$ is the single point $\{0\}$ when $n=0$, the sphere $\partial B_{1}$ when $n=1$ and the ball $B_{n}$ when $n>1$ [18, Proposition 4.1]. For $\pi$ a unitary irreducible representation of highest weight $n$, let $T_{n}$ denote the the Fourier transform of $j(X) \pi(\exp (X)$. Let $\delta_{B_{n}}$ be the Dirac measure of $B_{n}$ and let $\chi_{B_{n}}$ be the characteristic function of 
$B_{n}$. We note that $\frac{\partial}{\partial \nu} \chi_{B_{n}}=\delta_{B_{n}}$, where $\frac{\partial}{\partial \nu}$ is the derivative in the radial direction. In this context, Theorem 1.1 states that

$$
\operatorname{supp} T_{n} \subseteq B_{n+1}
$$

and

$$
\text { singsupp } T_{n}=\partial B_{n-1} \cup \partial B_{n+1},
$$

and that for the right choice of basis for $\mathfrak{s u}(2)$, the entries of $T_{n}$ are polynomials in the derivatives in the root, toral and radial directions applied to the measure $\chi_{B_{1}} * \chi_{B_{n}}$.

This is slightly more precise than [4], where it is shown that $\operatorname{supp} T_{n} \subseteq B_{n+1}$ and singsupp $T_{n} \subseteq \bigcup_{0}^{\left[\frac{n+1}{2}\right]} \partial B_{(n+1-2 k)}$, and that the entries are polynomial in the derivatives in the root, toral and radial directions applied to the measure $\chi_{B_{1}}^{*(n+1)}$.

\section{REFERENCES}

1. R. F. V. Anderson, The Weyl functional calculus, J. Functional Analysis, 4 (1969), 240-267. MR0635128 (58:30405)

2. D. Arnal and J. Ludwig, La convexité de l'application moment d'un groupe de Lie, J. Functional Analysis, 4 (1992), 256-300. MR.1160080 (93j:22013)

3. V. I. Arnold, Mathematical Methods of Classical Mechanics, Springer-Verlag, Berlin, 1980. MR0997295 (90c:58046)

4. F. Cazzaniga, Kirillov's formula for noncentral functions on SU(2), Rend. Sem. Mat. Univ. Politec. Torino, 50 (1992), 233-242. MR.1249464 (94m:22012)

5. A. H. Dooley and N. J. Wildberger, Global character formulae for compact Lie groups, Trans. Amer. Math. Soc., 351 (1999), 477-495. MR1638234 (99i:22013)

6. V. Guillemin and S. Sternberg, Convexity properties of the moment mapping, Invent. Math., 77 (1984), 533-546. MR0759258 (86b:58042a)

7. Harish-Chandra, Differential operators on a semisimple Lie algebra, Amer. J. Math., 79 (1957), 87-120. MR0084104 (18:809d)

8. B. Jefferies, The Weyl calculus for Hermitian matrices, Proc. Amer. Math. Soc., 124 (1996), 121-128. MR 1301032 (96d:47023)

9. M. S. Khalgui, Caractères des groupes de Lie, J. Funct. Anal., 47 (1982), 64-77. MR0663833 (84f:22020)

10. A. A. Kirillov, Characters of unitary representations of Lie groups, Funkcional. Anal. i Priložen, 2 (1968), 40-55. MR0236318 (38:4615)

11. B. Kostant, On convexity, the Weyl group and the Iwasawa decomposition, Annals sci. École norm. sup. (4), 6 (1973), 413-455. MR0364552(51:806)

12. J.-J. Loeb, Formule de Kirillov pour les groupes de Lie semi-simples compacts, Analyse harmonique sur les groupes de Lie (Sém., Nancy-Strasbourg, 1973-75), 230-256. Lecture Notes in Math., Vol. 497, Springer, Berlin, 1975. MR0407204 (53:10987)

13. K.-H. Neeb, Holomorphy and convexity in Lie theory, Walter de Gruyter \& Co., Berlin, 2000. MR:1740617 (2001j:32020)

14. E. Nelson, Operants: A functional calculus for non-commuting operators, Functional Analysis and Related Fields (Proc. Conf. for M. Stone, Univ. Chicago, Chicago, Ill., 1968), Springer, New York, 1970, pp. 172-187. MR0412857 (54:978)

15. L. Pukánszky, On the characters and the Plancherel formula of nilpotent groups, J. Functional Analysis, 1 (1976), 255-280. MR0228656 (37:4236)

16. W. Rossmann, Kirillov's character formula for reductive Lie groups, Invent. Math., 48 (1978), 207-220. MR0508985 (81g:22012)

17. R. Sjamaar, Convexity properties of the moment mapping re-examined, Adv. Math., 138 (1998), 46-91. MR1645052 (2000a:53148)

18. N. J. Wildberger, The moment map of a Lie group representation, Trans. Amer. Math. Soc., 330 (1992), 257-268. MR1040046 (92f:58064) 
MATRIX COEFFICIENTS AND COADJOINT ORBITS OF COMPACT LIE GROUPS 2571

School of Mathematics, University of New South Wales, Sydney NSW 2000, AusTRALIA

E-mail address: a.dooley@unsw.edu.au

School of Mathematics, University of New South Wales, Sydney NSW 2000, AusTRALIA

E-mail address: raed@maths.unsw.edu.au 